\title{
Building norms-adaptable agents from Potential Norms Detection Technique (PNDT)
}

\begin{abstract}
This paper presents a contribution to research on norms detection by proposing a technique, which is called the Potential Norms Detection Technique (PNDT). The literature proposes that an agent changes or updates its norms based on the variables of the local environment and the amount of thinking about its behaviour. Consequently, any changes on these two variables cause the agent to use the PNDT to update the norms in complying with the domain's normative protocol. This technique enables an agent to update its norms even in the absence of sanctions from a third-party enforcement authority as found in some work, which entail sanctions by a third-party to detect and identify the norms. The PNDT consists of five components: agent's belief base; observation process; Potential Norms Mining Algorithm (PNMA) to detect the potential norms and identify the normative protocol; verification process, which verifies the detected potential norms; and updating process, which updates the agent's belief base with new normative protocol. The authors then demonstrate the operation of the algorithm by testing it on a typical scenario and analyse the results on several issues.
\end{abstract}

Keyword: Intelligent software agent; Normative protocol normative system; Norms change; Norms detection; Norms mining 